\title{
Breve historia de la evolución en la oftalmología y del instituto de oftalmología «Fundación Conde de Valenciana»
}

\author{
Brief history of ophthalmology and "Conde de \\ Valenciana's Foundation» eye institute
}

\author{
David Lozano Rechy*
}

Citar como: Lozano RD. Breve historia de la evolución en la oftalmología y del instituto de oftalmología «Fundación Conde de Valenciana». An Med (Mex). 2020; 65 (4): 320-324. https://dx.doi.org/10.35366/97472

\section{RESUMEN}

La fragmentación de la medicina en diferentes especialidades ha promovido el desarrollo de mayor concentración del conocimiento en el arte de curar enfermedades. Éste ha sido el trayecto de la oftalmología al paso del tiempo, en el que se ha formado a su vez un grupo de 10 subespecialidades dedicadas al tratamiento de enfermedades que afectan a los ojos, y que han llevado a la construcción de hospitales dedicados exclusivamente al diagnóstico y tratamiento de problemas que afectan la visión y las estructuras que forman al sistema visual. El Instituto de Oftalmología Conde de Valenciana, que cumple 45 años de constante crecimiento, es muestra sólida de éxito del trabajo en equipo y que ha llevado a esta institución a ser orgullo de la medicina mexicana. Hoy en día, la fusión del Centro Médico ABC y la sede Santa Fe del Instituto Conde de Valenciana aporta a nuestra población atención oftalmológica de clase mundial.

Palabras clave: Oftalmología, instituto, historia, Conde de Valenciana.

Nivel de evidencia: III

\footnotetext{
* Director, Instituto de Oftalmología Conde de Valenciana, Profesor de Postgrado en Oftalmología, Universidad Nacional Autónoma de México, Profesor Titular de Oftalmología, Universidad Anáhuac, Miembro del Cuerpo Médico del Centro Médico ABC.
}

Recibido para publicación: 03/11/2020. Aceptado: 10/11/2020.

Correspondencia: David Lozano Rechy Chimalpopoca Núm. 14, Col. Centro, 06800 ,

Alcaldía Cuauhtémoc, CDMX, Tel: 55 5442-1700

E-mail: davidretina@gmail.com

\begin{abstract}
Fragmentation of medicine in different specialties has developed knowledge concentration in the art of curing diseases. This is the story of ophthalmology along time, where ten subspecialities have been formed to treat eye diseases. This has led at the same time, to the building of eye hospitals dedicated exclusively to the diagnosis and treatment of problems that affect vision and structures related to the visual system. In 2021, Conde de Valenciana Eye Institute will celebrate its $45^{\text {th }}$ anniversary of constant growing, which represents a national pride as an example of solid success of teamwork in Mexican medicine. In the present time, the fusion of Conde de Valenciana Eye Institute and $A B C$ Medical Center represents a world class ophthalmology service to our population.
\end{abstract}

Keywords: Ophthalmology, institute, history, Conde de Valenciana.

Level of evidence: III

La oftalmología es probablemente tan antigua como la necesidad de ver. El intentar curar las enfermedades que afectan a los ojos ha sido una práctica de todas las culturas y ha evolucionado como otras especialidades médicas dentro de un proceso de fragmentación y síntesis. Así es como han ido apareciendo las llamadas subespecialidades y grupos de especialistas dedicados a determinadas áreas de la medicina. La oftalmología hoy en día es probable- 
mente la especialidad con más subespecialidades en la práctica médica. Prueba de ello es el claro crecimiento en el número de hospitales dedicados exclusivamente a la atención de personas que padecen enfermedades de los ojos.

El Instituto de Oftalmología «Fundación Conde de Valenciana» es una institución de asistencia privada que no persigue fines de lucro. Existe gracias al legado de Don Luis Ludert y Rul (1868-1953) (Figura 1), descendiente de los Condes de Valenciana (Figura 2), quién fue sin duda un hombre orgulloso de su familia y de su linaje, lo que explica el porqué, al crear la fundación, omitió darle su nombre y en su lugar dio origen a la «Fundación Conde de Valenciana» en recuerdo de sus antepasados. ${ }^{1}$

Don Luis se casó a una edad madura y su matrimonio duró poco, sin descendencia. Vivió sus últimos años en una casona señorial en la calle de Ezequiel Montes de la Ciudad de México, donde conoció la necesidad de brindar atención oftalmológica a las personas de escasos recursos y porque servir al pueblo era su voluntad. ${ }^{2}$

A su muerte dejó un predio en la calle de Chimalpopoca esquina con Niño Perdido (hoy Lázaro Cárdenas) en la misma Ciudad de México, propiedad donde

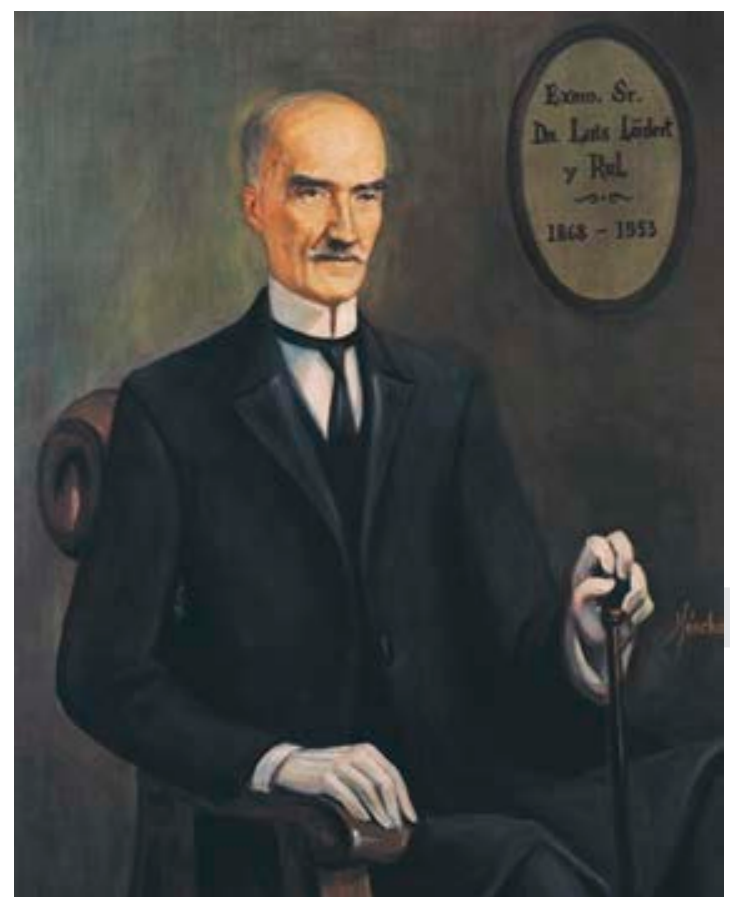

Figura 1: Retrato de Don Luis Ludert y Rul, quien legó el patrimonio de la Fundación Conde de Valenciana. Obra museográfica de la Fundación Conde de Valenciana. I.A.P.

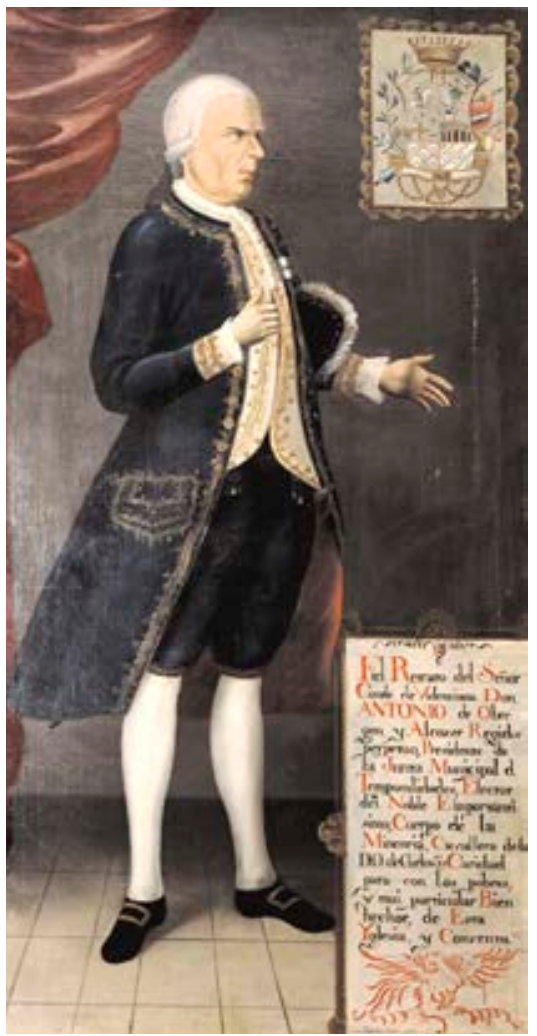

Figura 2:

Retrato del Señor Conde de Valenciana Don Antonio de Obregón y Alcocer, regidor perpetuo, presidente de la Junta Municipal de Temporalidades, elector del Noble e Importantísimo Cuerpo de Minería, Caballero de la D.O. de Carlos III. Obra museográfica de la Fundación Conde de Valenciana. IAP.

hoy está asentada la institución por ser una colonia popular y céntrica así como recursos suficientes para la construcción de un hospital que se dedicara a la atención de enfermos de los ojos, especialmente de aquéllos de escasos recursos. ${ }^{2}$

El hospital abrió sus puertas al público en febrero de 1976, la construcción contaba con una torre de hospitalización y una unidad de consulta externa ( $F i$ gura 3). Los primeros años de actividad fueron económicamente difíciles, pues aunque el hospital crecía en servicio, requería de flujos de capital, de los cuales se carecía. La dificultad económica y la dificultad que representó la utilización de los inmuebles donados por Don Luis, deteriorados y conflictivos o expropiados por el gobierno, llevaron al Dr. Enrique Graue y Díaz González -albacea del legado de Don Luis- a la creación de un patronato, el cual hoy en día depende de una organización oficial llamada «Junta de Asistencia Privada». Desde un principio, el papel del patronato ha sido esencial por sus esfuerzos para rescatar parte del legado y acrecentar el capital destinado a la creación de un instituto. ${ }^{2}$

El Patronato del Instituto de Oftalmología «Fundación Conde de Valenciana» mantiene esta obra de beneficio social gracias a los recursos obtenidos a 
An Med (Mex) 2020; 65 (4): 320-324

través de cuotas de recuperación, las que se procura mantener siempre a los precios más bajos posibles. Los ingresos que se obtienen por las cuotas de recuperación se emplean cabalmente en el mantenimiento de las instalaciones, en el pago al personal, en la adquisición de tecnología de vanguardia y en apoyar con servicios médicos y quirúrgicos gratuitos a quien, por su condición económica, no puede cubrir los costos de su atención. La gran mayoría de personal médico, incluyendo a todos sus directores, ha trabajado desde entonces en forma voluntaria sin remuneración económica.

En el intento de conquistar la excelencia, la Fundación Conde de Valenciana decidió respaldarse en la enseñanza, renglón en el que el instituto ha logrado un liderazgo, por el que hoy lo convierte en una de las instituciones más reconocidas y solicitadas para ser sede de los cursos de pregrado de distintas universidades así como del Postgrado en Oftalmología de la Universidad Nacional Autónoma de México con la residencia en esta especialidad de la medicina. El Instituto Conde de Valenciana ha sido creador de múltiples cursos y entrenamiento en alta especialidad en las áreas de córnea y cirugía refractiva, imagenología ocular y orbitaria, órbita, párpados y vías lagrimales, retina y vítreo, microcirugía del segmento anterior del ojo, estrabismo, glaucoma, enfermedades de la úvea, rehabilitación visual y oftalmogenética; a su vez, se ha creado la Maestría y Doctorado en Ciencias Médicas y la Maestría Binacional en Ciencias de la Visón para alumnos no sólo mexicanos, sino de otros países. ${ }^{3}$

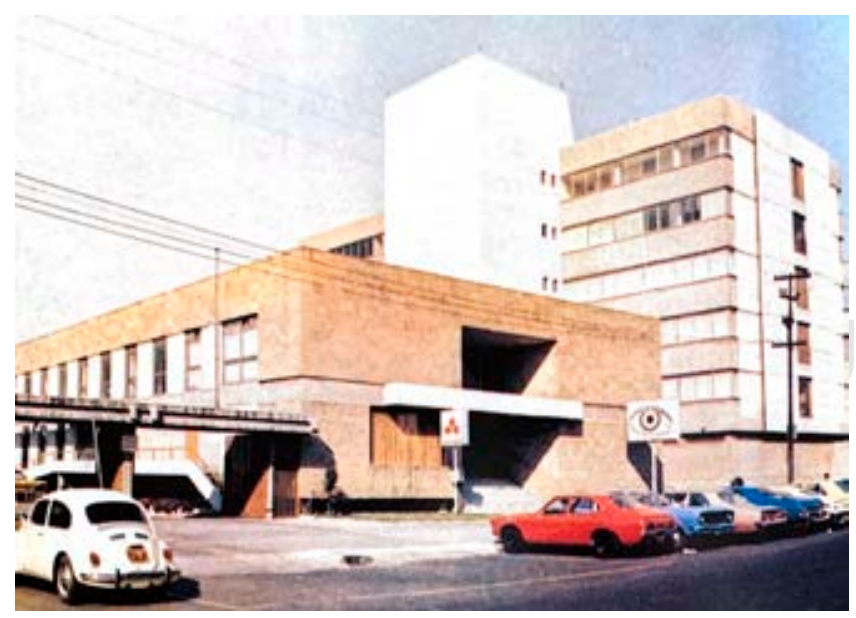

Figura 3: Fotografía del primer edificio de la Fundación Conde de Valenciana constituido por una torre de hospitalización y una unidad de consulta externa, inaugurado en 1976.

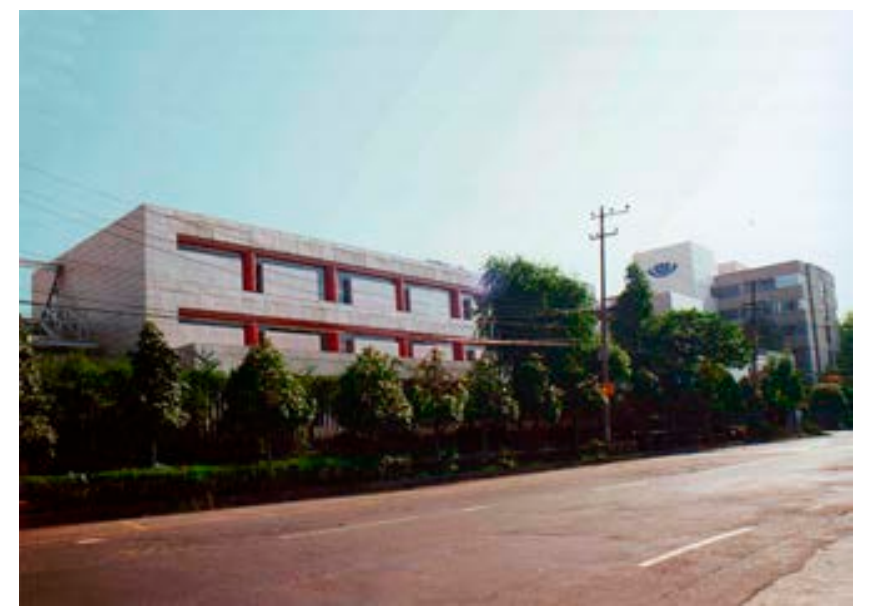

Figura 4: Fotografía del segundo edificio de la fundación, con remodelación del primer edificio, donde se instaló la unidad de investigación, inaugurada en el año 2000.

Posterior a dos décadas, el instituto desarrolló un incremento vertiginoso en su reconocimiento internacional debido a la creación de su unidad de investigación (Figura 4) y al respaldo científico que proporciona en las áreas de biología molecular, genética, inmunología y microbiología asociadas a enfermedades de los ojos. Es en ese espacio donde se crean los conceptos evolutivos de los conocimientos especializados y que posteriormente se aplican en la clínica, lo que ha derivado en aportaciones de alta calidad científica en el conocimiento de enfermedades oculares, trasplante de tejidos y procedimientos quirúrgicos que han beneficiado a un número indescriptible de pacientes. Gracias a la unidad de investigación en trabajo de equipo con los departamentos de sus 11 subespecialidades en oftalmología, el Instituto Conde de Valenciana se coloca entre los primeros cuatro lugares de instituciones de investigación oftalmológica en América Latina. ${ }^{4}$

Durante más de 44 años el hospital no sólo ha sido testigo, sino protagonista de una parte de la historia de un mundo que vive en transformaciones a pasos agigantados, mismas que han permitido el desarrollo de recursos humanos y tecnológicos que producen cambios en la vida de las instituciones de salud. A la par de este progreso, el instituto ha superado las metas propuestas. A pesar de que en algunos momentos se ha sufrido para hacer frente a las lamentables crisis económicas, a las dificultades de inseguridad y violencia que vivimos, a las crisis ocasionadas por los temblores de 1985 y 2017 así como por las crisis sanitarias por la influenza AH1-N1 y COVID-19, todo ello ha 
provocado un incentivo extraordinario para superar sus consecuencias gracias al espíritu que emergió en cada uno de los miembros de su comunidad.

Entre 1976 y 2005 el instituto dedicó sus esfuerzos a atender los problemas de salud visual de quienes habitan o logran llegar a la Ciudad de México, sin menoscabo del lugar de residencia, condición social o situación económica, pero dejando fuera a muchos más.

Por ello, en 2004 se decidió enfrentar un nuevo reto: llevar a la comunidad de mayor rezago social los servicios oftalmológicos de excelencia; para ello y con el apoyo de Nacional Monte de Piedad IAP, la Fundación Gonzalo Río Arronte IAP y otros donantes anónimos se construyó en una zona de alta

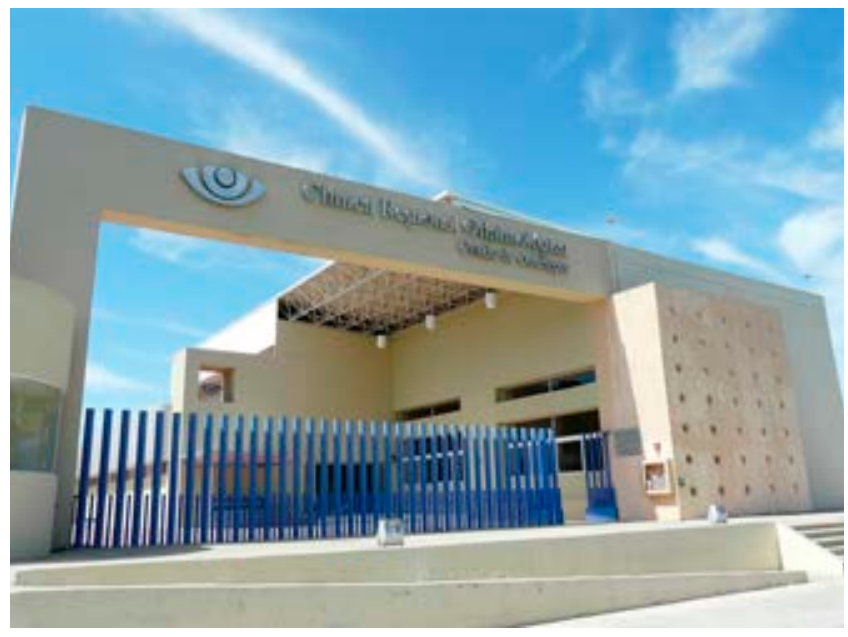

Figura 5: Fotografía de la sede Conde de Ometepec en una de las zonas marginadas del país, en la costa chica del estado de Guerrero, inaugurada en 2005 .

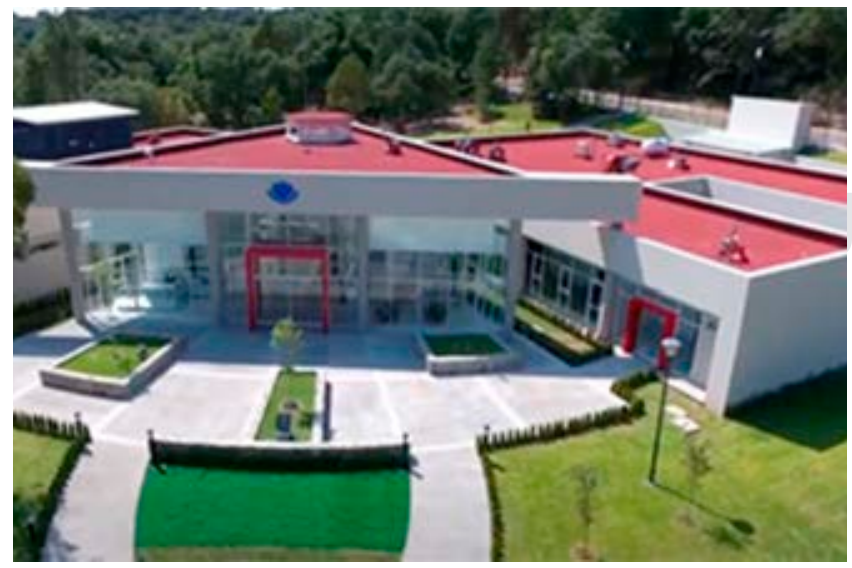

Figura 6: Fotografía de la sede en el estado de Tlaxcala, inaugurada en 2017.

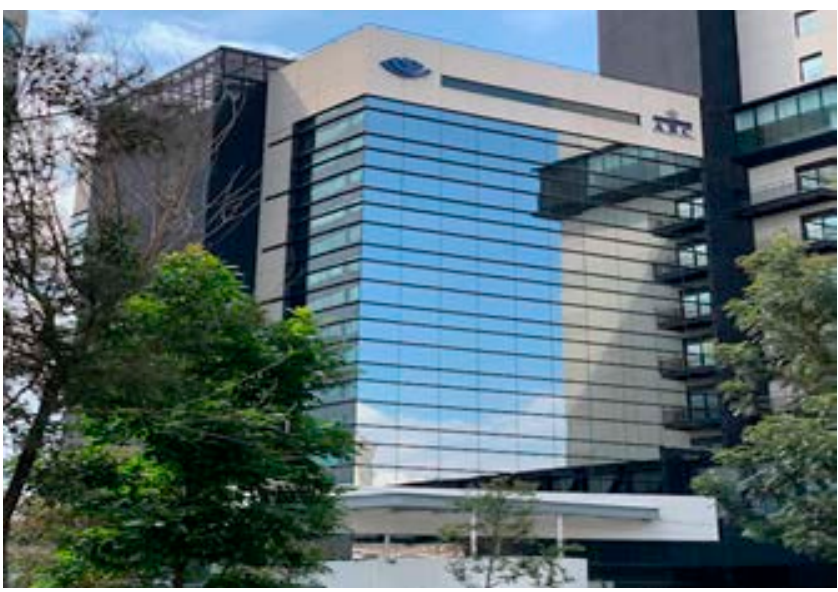

Figura 7: Fotografía de la sede en el edificio colindante con el Centro Médico ABC, campus Santa Fe, inaugurada en 2017.

marginación y población indígena del estado de Guerrero, la Clínica Regional Oftalmológica Conde de Ometepec (Figura 5), que de 2005 a la fecha ha beneficiado a más de 150,000 personas. En el mismo sentido, el instituto inauguró en 2017 la sede Tlaxcala (Figura 6) en la zona de hospitales de la localidad de San Matías Tepetomatitlán, Municipio de Apetatitlán, donde hoy se han llevado a cabo más de 70,000 consultas y más de 7,000 cirugías oftalmológicas. Desde 2017 también y gracias a la excelencia en el tratamiento integral de padecimientos visuales y al prestigio nacional e internacional, el Conde de Valenciana Sede Santa Fe ofrece sus servicios privados de alta especialidad en oftalmología en alianza con el Centro Médico ABC (Figura 7), al que se incorporó como parte integral del servicio de oftalmología de este prestigiado centro médico, con la cultura de práctica médica grupal y evaluado con las normas más estrictas de seguridad del paciente a través de la Joint Commission International.

La atención de más de 100,000 pacientes y más de 10,000 cirugías oftalmológicas anuales, la unidad de investigación, el papel social de la fundación en la gestión del Banco de Ojos de la Cruz Roja Mexicana-Instituto de Oftalmología, las campañas de cirugías extramuros, el Centro de Rehabilitación para Ciegos y Débiles Visuales así como la creación de cuatro sedes en diferentes zonas del país hacen que la institución sea una parte esencial de la historia de la oftalmología mexicana en el último cuarto del siglo XX y en el primero del siglo XXI, contribuyendo así con la generación de cultura de salud visual que tiene repercusiones positi- 
An Med (Mex) 2020; 65 (4): 320-324

vas en la calidad de vida de las personas durante sus diferentes etapas.

Con esta combinación de buenas voluntades y módicas cuotas, a casi medio siglo de su fundación, la Fundación Conde de Valenciana es una institución oftalmológica que se cuenta entre las más prestigiosas de América Latina, y sin duda una de las más grandes. La calidad de sus servicios y la cuantía de éstos en conjunto con modernas instalaciones y equipos de vanguardia hacen de la institución un motivo de reconocimiento nacional e internacional.

\section{REFERENCIAS}

1. Graue WE. XXV Aniversario Instituto de Oftalmología Fundación Conde de Valenciana. Editorial Laser, S.A. de C.V. Imp. México D.F. 2001, pp. 51-109.

2. Graue WE. XXV Aniversario Instituto de Oftalmología Fundación Conde de Valenciana. Editorial Laser, S.A. de C.V. Imp. México D.F. 2001. pp. 111-119.

3. Cano HR. Treinta y cinco años de vocación científica. Instituto de Oftalmología Fundación Conde de Valenciana. $2^{\mathbf{a}}$. ed. de; Aniversario de la Fundación Conde de Valenciana. Editorial Castellanos Editores, SA de CV, José Luis González Olalde. Imp. México D.F., 2011.

4. SCOPUS de Elsevier 2020. Available in: https://www. scopus.com/. 